\title{
L'entretien d'explicitation : une approche potentiellement féconde pour faciliter la supervision clinique des résidents
}

\section{The clarification interview: a potential approach to improve clinical supervision of residents}

\section{Thierry LIEVIN ${ }^{1}$, Marquis FORTIN², Bernard MILLETTE ${ }^{2}$, Alain AUBREGE ${ }^{1}$ et Jean-Dominique De KORWIN ${ }^{1}$}

1 Département universitaire de médecine générale, Faculté de médecine, Université Henri Poincaré, Nancy, France

2 Département de médecine familiale, Faculté de médecine, Université de Montréal, Montréal, Québec, Canada

Manuscrit reçu le 5 mai 2005 ; commentaires éditoriaux formulés aux auteurs le 13 juillet 2005, le 15 septembre 2006 et le 30 septembre 2008 ; accepté pour publication le 6 octobre 2008

\section{Mots clés :}

Formation initiale ; médecine générale; médecine familiale ; entretien d'explicitation ; entretien pédagogique

\section{Keywords:}

Post-graduate training; general practice; family medicine; clarification interview; educational interview
Résumé - Contexte : L'entretien occupe une place importante durant la supervision clinique du résident; il comporte des limitations qui peuvent être corrigées. But : Faire le point sur l'entretien pendant la supervision du résident, réfléchir sur ses insuffisances, et proposer une méthode d'amélioration. Méthode : 1) Expérience pratique de formation clinique et pédagogique lors d'un stage de 8 mois de résidanat en Unité de Médecine Familiale (UMF) et de 3 mois de préceptorat à l'université de Montréal (Canada);2) revue de la littérature centrée sur la formation initiale en soins primaires, sur la communication, et sur les techniques de verbalisation en psycho-ergonomie. Résultats : L'observation de la formation en UMF et la revue de la littérature, pointent l'importance de l'entretien dans la supervision clinique du résident. Ses insuffisances comprennent : perte d'opportunité de renforcer l'apprentissage, défaut d'organisation, mode de conduite et formulation inadaptée des questions et des relances, substitution de connaissances. Elles amoindrissent la performance de l'enseignement. Nous pensons que l'entretien d'explicitation apporte des réponses à ces problèmes. Cette méthode intègre des techniques communicationnelles spécifiques. Elle favorise le développement des savoirs expérientiels, et évite les biais faussant l'entretien en situation de supervision. Conclusion : L'entretien d'explicitation ouvre des perspectives intéressantes pour améliorer la formation du résident. Il trouverait sa place dans un tutorat personnalisé. Il nécessite une formation spécifique.

Abstract - Background: The interview holds an important place during residents' clinical supervision; it comprises limitations that can be improved. Purpose: To review the interview during residents' supervision, to think on its limitations and to propose a method of improvement. Method: 1) Clinical training and educational experiences during a training course of 8 months of residency in a Family Medicine Unit (FMU) and a training course of 3 months of tutorship at the University of Montreal (Canada); 2) Literature review based on primary 
care post-graduate training, communication and psycho-ergonomics verbalisation methods. Results: The observation of training in FMU and the review of the literature pointed out the importance of the interview during the clinical supervision of residents. Its limitations comprise: a loss of opportunity to improve learning, a deficiency in organization, an unsuited mode of control, an unsuited formulation of questions and revivals and a substitution of knowledge. These limitations weaken the efficiency of teaching. We think that the clarification interview brings about solutions to these problems. This method integrates specific communication techniques. It supports the development of experiential knowledge and avoids bias that distorts the interview in the context of supervision. Conclusion: The clarification interview opens interesting perspectives to improve residents' clinical training. It could be addressed for personalized tutoring. It needs a specific training.

\section{Introduction}

Selon Kilminster et al. ${ }^{[1,2]}$, la supervision clinique consiste en la fourniture d'une guidance à l'égard d'un étudiant en formation et d'une rétroaction (feedback) à l'égard de son développement personnel, professionnel et éducationnel, dans le contexte d'une expérience de soins dispensés à un patient avec sécurité et de manière appropriée. Comme le rappellent Martineau et al. ${ }^{[3]}$, elle peut revêtir plusieurs modalités. Ainsi, la procédure peut en être formelle ou informelle et elle peut selon les cas être associée à une observation directe de l'étudiant en situation de pratique et être ainsi immédiate ou, au contraire, être différée lorsque le superviseur n'a pas assisté à la situation clinique et qu'il fournit sa rétroaction dans un second temps, à partir de la présentation qu'en fait l'étudiant.

Dans ce dernier cas, l'entretien entre le résident (ou interne) et l'enseignant responsable de la supervision clinique occupe une place centrale puisque c'est à ce moment, à partir d'un rapport verbal, que le superviseur évalue la qualité de la prise en charge du patient et que le résident reçoit des informations sur son atteinte des objectifs de sa formation. Souvent, cet entretien est limité à l'appréciation d'un résultat professionnel et vise essentiellement la sécurité des patients, ou bien il est exploité systématiquement et méthodiquement pour développer et orienter les apprentissages à effectuer par les étudiants supervisés.

Or, nous pensons que cet entretien, dans le but d'en améliorer l'efficience à la fois clinique et pédagogique, devrait être également l'opportunité d'évaluer un processus d'apprentissage, spécifique à chacun, et d'initier un « réfléchissement ». Nous employons ce terme dans l'acception que lui a attribuée Vermersch, pour désigner une prise de conscience provoquée à partir d'un vécu d'action pendant un entretien, au cours duquel l'intervieweur « reflète» ce vécu pour susciter cette prise de conscience, en agissant comme un miroir tourné vers l'interviewé. Dans cette perspective, l'organisation spécifique des conditions de l'entretien de supervision et l'entraînement des enseignants à certaines techniques d'entretien, en l'occurrence l'entretien d'explicitation, ouvrent des perspectives d'amélioration de la formation du résident.

À partir d'une étude systématique de la littérature, complétée par une expérience personnelle d'observation participante, nous proposons une analyse des problèmes posés par l'entretien de supervision puis, dans un deuxième temps, nous décrivons les bases d'un entretien codifié, l'entretien d'explicitation, qui constitue à nos yeux une piste appropriée pour apporter des solutions aux problèmes identifiés.

\section{Méthodes}

Notre analyse des problèmes posés par l'entretien au cours de la supervision clinique des résidents (ou internes) a été élaborée à partir de deux démarches : a) une revue méthodique de la littérature consacrée à la problématique de la communication résident-enseignant dans le contexte de la formation 
en médecine générale et aux techniques d'entretien applicables à la formation professionnelle; b) une expérience d'observation participante de la formation des résidents conduite par l'un d'entre nous dans une unité de médecine familiale associée à l'université de Montréal, dans le cadre d'un stage de formation en pédagogie, organisé par l'unité de recherche et de développement en éducation des sciences de la santé (URDESS) de cette université.

\section{Revue méthodique de la littérature}

Notre revue méthodique de la littérature a comporté une recherche documentaire informatisée, complétée par une recherche manuelle effectuée à partir des ressources bibliographiques des structures suivantes : le département de médecine familiale de l'université de Montréal (DMFUM); l'unité de recherche et de développement en éducation des sciences de la santé (URDESS) de l'université de Montréal); le laboratoire de psychologie de l'homme au travail de l'institut national de recherche et de sécurité (INRS) de Nancy.

La recherche documentaire informatisée a exploité les ressources disponibles sur la base de données bibliographiques Medline, interrogée en ligne grâce à l'interface «PubMed ». Le protocole de recherche a porté sur le titre des documents indexés (Search «Key words » Field : Title), à partir de la liste de mots clés suivants : "teaching learning ambulatory setting », " teaching learning ambulatory settings ", "medical education primary care », "medical education ambulatory setting », "medical education ambulatory settings », "improving teaching skills», "resident physician interactions », "communication primary care », "communication resident physician", "communication student physician », "interview residents », «interview residents physicians », "interview students», "interview students physicians », "interview students faculty ». Les articles ainsi sélectionnés ont fait l'objet d'une première analyse à partir de la lecture des résumés. Ont été conservés les articles dont l'objet portait explicitement sur : la formation initiale en médecine générale; la communication en médecine générale. Lorsque la liste de réponse dépassait un seuil de 40 articles, seuls les articles de synthèse étaient conservés. Les types d'articles suivants ont été exclus : articles sans mention d'auteurs, articles ciblant une catégorie spécifique de problèmes médicaux, articles sans résumés (à l'exception de deux références déjà en possession des auteurs, qui ont été gardées malgré l'absence de résumé : Lawrence RS. Medical education in ambulatory setting. Arch Intern Med 1990 ; 150 : 2008-2009. Lawrence RS. The goals for medical education in the ambulatory setting. J Med Intern Gen 1988 ; 3 : S15-S25.), articles portant sur la formation « undergraduate » (équivalent du deuxième cycle des études médicales) sans rapport évident avec les soins primaires ou la communication professionnelle.

La recherche documentaire informatisée effectuée en ligne a été complétée par une recherche non systématique à partir des articles et des ouvrages prêtés par le DFMUM, par l'URDESS, et par l'INRS. Les références concernant l'approche dite de «Calgary-Cambridge » et certains documents sur les travaux de DA Schön, ont été proposés par le DMFUM. Les références prêtées par l'INRS concernaient des techniques de verbalisation en psychoergonomie, dont l'entretien d'explicitation. L'URDESS a proposé des documents sur les travaux de DA Schön.

\section{Observation participante au sein d'une unité de médecine familiale}

L'un des auteurs (TL) a suivi une formation complémentaire à l'université de Montréal à la fin de son résidanat de médecine générale. L'université Henri Poincaré (faculté de médecine) de Nancy (France), le centre hospitalier et universitaire de Nancy et l'université de Montréal, ont organisé cette formation, qui incluait : un stage clinique (8 mois) comme résident de première année dans une unité de médecine familiale (UMF) ; un stage de pédagogie médicale (3 mois) dans le cadre d'un préceptorat proposé par l'URDESS. 
Les objectifs de chaque stage étaient respectivement d'observer et de faire l'expérience de la formation initiale en médecine générale organisée par la faculté de médecine de l'université de Montréal et développer les connaissances nécessaires à l'appréciation des méthodes pédagogiques observées au cours du stage clinique. Durant cette expérience, la collecte de données sur les stratégies et techniques d'enseignement développées dans le milieu observé a été conduite sous diverses formes : observation et prise de notes régulières, discussion avec les responsables de la formation, discussion avec les responsables du stage de pédagogie.

Le programme hebdomadaire de formation à l'UMF était réparti sur plus de 20 heures selon les proportions suivantes : $45 \%$ à $75 \%$ de consultations autonomes supervisées auprès des patients dans la première moitié du stage, et $75 \%$ dans la dernière moitié ; jusqu'à $30 \%$ d'observation de consultations données par des médecins de famille dans la première moitié du stage, activité remplacée ensuite par des consultations autonomes supervisées; $15 \%$ de discussions collégiales supervisées sur le contenu des consultations ; $10 \%$ d'activités de formation à la communication médecin-patient.

Le programme du préceptorat comprenait l'équivalent de 43 heures de leçons, 267 heures de travaux pratiques, huit heures d'ateliers, de nombreuses heures de lecture et de synthèse. Il se concluait par la rédaction d'un mémoire. Cet agenda a été complété par cinq journées de séminaires portant sur les thèmes de la rétro-action (feedback) et du «réfléchissement ».

\section{Résultats}

Les problèmes posés par l'entretien de supervision

\section{Les données issues de la revue de la littérature}

La procédure de recherche documentaire informatisée en ligne permettait de limiter la recherche à une littérature spécifiquement consacrée à la communication et à la formation en soins primaires. Nous voulions éviter tant la dispersion du contenu des articles retrouvés, que la collection d'une quantité énorme de documents, dont la revue serait trop longue et trop complexe. Les articles sélectionnés étaient tous consacrés spécifiquement à la médecine générale, à la médecine interne générale, à la communication médicale professionnelle ou à l'éducation médicale. Cette bibliographie ne reflète exactement l'importance ni de la littérature consacrée à la formation initiale en soins primaires, ni de celle consacrée à la communication enseignant-résident. Les critères de sélection choisis ont abouti à exclure des articles malgré la mention des mots-clés recherchés dans leur titre. L'importance de la littérature concernée est donc potentiellement plus grande que celle sélectionnée dans ce travail. Un total de 20 références, publiées entre 1980 et 2002, ont été sélectionnées sur internet : 18 articles et deux revues systématiques de la littérature.

\section{L'entretien entre le résident et l'enseignant superviseur}

L'entretien dédié à la supervision clinique entre un enseignant et un étudiant (résident ou interne) est mentionné dans huit articles ${ }^{[6-13]}$, parmi lesquels une revue systématique de la littérature ${ }^{[7]}$. Il est décrit comme très court ${ }^{[7,9,14]}$, avec un abord superficiel des dimensions pédagogiques ${ }^{[7,9]}$; c'est un mode de supervision très courant en contexte de soins primaires ${ }^{[13]}$.

Plusieurs recommandations sont formulées : 1) élaborer et développer un climat de confiance entre l'étudiant et l'enseignant ${ }^{[6,8,11,12]}$;2) explorer avec neutralité et objectivité les connaissances médicales acquises par l'étudiant en lien avec le contenu médical abordé ${ }^{[6,8,11]}$; 3) explorer avec neutralité et objectivité la représentation développée par l'étudiant des problèmes médicaux rencontrés ${ }^{[6,8,12]}$; 4) faire participer l'étudiant à un «réfléchissement» sur l'action conduisant à mettre en rapport ses représentations subjectives des problèmes rencontrés avec les connaissances nécessaires à la compréhension du contenu abordé ${ }^{[6,8,10,12]}$. 


\section{La communication en santé}

Les articles colligés portent surtout sur la communication médecin-patient en soins primaires ${ }^{[15-19]}$. Deux autres s'intéressent à la relation résidentenseignant ${ }^{[9,14]}$. Deux autres, du même auteur, suggèrent que la prise en charge pédagogique de l'étudiant et l'éducation sanitaire du patient sont comparables en matière de stratégie communicationnelle ${ }^{[6,8]}$.

Plusieurs recommandations sont également formulées : 1) une formation dédiée à une meilleure compréhension à la fois des comportements verbaux et non verbaux améliore la communication et devrait être encouragée ${ }^{[15-17]}$; 2) la sensibilisation au processus de l'entretien médical est importante pour le développement des habiletés communicationnelles ${ }^{[19]}$; 3) l'enseignement et l'évaluation devraient être centrés sur une vision large de la communication en médecine ${ }^{[19]}$; 4) l'enseignement des habiletés communicationnelles et cliniques devrait être cohérent et complémentaire $^{[19]}$; 5) l'enseignement devrait définir des objectifs de communication centrés sur le patient $^{[19]}$ et aider les étudiants à les atteindre.

\section{L'approche "Calgary-Cambridge »}

Cette approche de la communication professionnelle ${ }^{[20]}$ procède directement des résultats de la recherche des 40 dernières années auprès de populations de patients très divers. Ses objectifs comprennent : la promotion de la collaboration et du partenariat dans l'action; l'amélioration de la performance médicale ; l'amélioration de la satisfaction des protagonistes de l'action.

Quatre principes soutiennent l'approche «Calgary-Cambridge » ${ }^{[20]}:$ 1) la communication professionnelle est une compétence essentielle à l'atteinte des objectifs de soins primaires; 2) elle procède de compétences qui peuvent être enseignées et apprises ; 3) l'amélioration de la pratique a besoin d'un feedback spécifique, sans lequel elle devient difficile et plus imparfaite; 4) la maîtrise des habiletés communicationnelles nécessite un entraînement régulier et spécial.
Plusieurs conditions assurent l'efficacité de la communication et de l'enseignement ${ }^{[20]}$ : le maintien d'une interaction réciproque durant l'entretien; la prise en compte de la gestion de l'incertitude; l'accent mis sur la capacité d'adaptation aux changements; la référence à un modèle de progression hélicoïdale, mieux adapté à la gestion de l'inattendu au cours des entretiens.

L'entretien professionnel selon l'approche «Calgary-Cambridge » implique, entre autres, le co-déroulement de deux processus ${ }^{[20]}$. D'une part, la structuration de l'entretien suppose d'en rendre explicite l'organisation et de prêter attention à son déroulement. D'autre part, la construction de la relation implique d'utiliser des comportements verbaux et non verbaux adaptés, d'établir une ambiance conviviale et d'associer l'interlocuteur à la démarche professionnelle. Des habiletés spécifiques sont utilisées au cours de ces processus : gestion des questions ouvertes ou fermées, écoute active, gestion des silences, énoncé de soutien, signes de compréhension, répétitions en écho, reflets, paraphrases, etc. L'attention portée au comportement non verbal de l'interlocuteur apporte des informations utiles sur le rapport qu'il établit avec son récit. Les clarifications et les synthèses du récit entendu rythment l'élucidation du problème rapporté par l'interlocuteur. Les clarifications s'attachent à expliciter les généralisations en obtenant les faits réels qui en sont à l'origine, à déceler les omissions, les sous-entendus ou les présupposés, à lever les ambiguïtés. Les synthèses permettent à l'interlocuteur de vérifier la compréhension qu'a l'intervieweur du problème exploré.

\section{Les travaux de Schön}

Schön a modélisé la pratique professionnelle, toutes disciplines confondues, d'après des observations rapprochées et prolongées, réalisées dans les années $1980^{[21]}$. Il en décrit trois composantes : «le savoirdans-l'action », "la réflexion pendant l'action» et «la réflexion après l'action»; l'ensemble des trois caractérise la «pratique réfléchie »-ou réflexive -. Celle-ci représente la démarche optimale de tout professionnel en exercice. 
Schön remarque que l'activité quotidienne d'un professionnel consiste à résoudre de problèmes complexes, spécifiques, mal définis, impliquant parfois des éléments contradictoires. Pour élaborer une solution face aux problèmes abordés en pratique, le professionnel dispose d'un répertoire d'exemples, de schèmes, d'une typologie des problèmes acquise par expérience et auxquels il confronte les situations rencontrées. Les interprétations de la situation qui en résultent sont ensuite éprouvées dans une démarche hypothético-déductive, dont le professionnel observe l'évolution et les résultats. Si certains problèmes sont résolus automatiquement, d'autres troublent les repères habituels du praticien, qui doit reconsidérer de façon critique ses procédures, pour les ajuster à la situation rencontrée. Cet ajustement passe par un retour réflexif du praticien sur sa propre démarche, qui permet de la corriger ou de l'enrichir, de manière à y intégrer les données nouvelles ${ }^{[21]}$.

Le «savoir-dans-l'action » s'exprime à l'instant d'une action professionnelle spécifique par un comportement efficace, automatique et spontané, sans intervention évidente d'une démarche intellectuelle réfléchie ${ }^{[21]}$, ce qui signifie que le praticien effectue ces différentes actions sans nécessairement en avoir conscience, ni savoir «comment il fait» (on parle de savoir tacite). La "réflexion pendant l'action» aboutit à l'ajustement durant l'action des procédures habituelles du praticien au cas particulier rencontré, alors que celui-ci est momentanément perturbé par une situation inédite ${ }^{[21]}$. La "réflexion après l'action » aboutit à l'ajustement de ces mêmes procédures à la même catégorie de cas rencontrés, mais consécutivement à l'action et par le biais d'un enrichissement, ou d'une modification a posteriori, permettant l'intégration des données nouvellement apprises. Ces composantes de la "pratique réfléchie »-ou réflexive - sont, selon Schön, cruciales pour le développement, le renouvellement et l'autocorrection de la compétence des professionnels ${ }^{[21]}$.

\section{Les biais des entretiens consécutifs à l'action}

La littérature sur la conduite d'entretien en psychoergonomie identifie des situations et des phénomènes aboutissant à fausser l'appréciation de l'intervieweur sur la démarche d'action rapportée par l'interviewé. Les principaux biais relevés sont les suivants :

- verbalisation d'opinions naïves sur l'action en substitution des connaissances réellement utilisées dans l'action : l'opérateur rapporte l'idée qu'il se fait de sa tâche et non les actes ou les raisonnements réellement effectués ${ }^{[22,23]}$;

- variation de la réponse de l'interviewé selon le mode de présentation de la consigne de verbalisation $^{[22]}$;

- variation de la réponse de l'interviewé selon la cible à laquelle s'adresse la consigne ${ }^{[16]}$ (les destinataires présumés

- néophyte ou expert -, l'utilisation ultérieure qui en sera faite);

- variation de la réponse selon l'information (ou la formation) préalable du sujet ${ }^{[16]}$ : le supervisé est-il informé qu'il sera supervisé ? En a-t-il l'habitude?

Plusieurs situations comportent ainsi un haut risque d'aboutir à la verbalisation d'un contenu satellite de l'action (contexte, théories, etc.) et non de savoirs réellement utilisés dans l'action (savoirs tacites) : verbalisation d'action hors guidance adaptée $^{[22,23]}$; verbalisation de procédures devenues automatiques ${ }^{[2,24]}$; verbalisation de procédures d'acquisition récente (connaissance encodée de manière conceptuelle et déclarative) ${ }^{[22,24]}$.

\section{Les données issues de l'observation participante en unité de médecine familiale}

Les résidents en médecine familiale du Québec sont formés dans des structures de soins ambulatoires intégrées à des hôpitaux ou à des centres communautaires, les unités de médecine familiale (UMF). Le stage clinique a permis d'observer des méthodes pertinentes et efficaces pour la formation des résidents en médecine familiale. Mais, durant l'année et dans le milieu où fut effectué le stage, les conditions de travail des médecins enseignants en supervision courante étaient difficiles compte tenu de la réduction récente des effectifs médicaux de l'UMF 
locale. En moyenne, cinq à six résidents arrivaient simultanément en salle de supervision, toutes les 15 à 20 minutes. En moyenne, deux superviseurs étaient disponibles et pouvaient consacrer cinq à huit minutes d'entretien par résident. Lorsqu'un seul superviseur était accessible, ce temps était réduit à deux à trois minutes par résident. Le superviseur était régulièrement confronté à des demandes différentes, conflictuelles, simultanées, qu'il devait gérer rapidement. L'enseignement était court et superficiel. Le feedback immédiat était de ce fait bref et relativement superficiel; il portait sur la pertinence des propositions du résident selon les critères du superviseur, que ce dernier étayait par des références bibliographiques nationales. L'exploration du processus par lequel le résident avait développé sa représentation du cas clinique était rare. La démarche visant à susciter un « réfléchissement » personnalisé, destiné à mettre en relation les connaissances nécessaires au traitement du problème médical abordé et la représentation élaborée par le résident, était rare.

L'interprétation de la qualité de la démarche clinique du résident procédait du récit entendu. Les biais d'appréciation se rencontraient à ce niveau. Certains superviseurs se distinguaient par la mâ̂trise de techniques d'entretien permettant d'aller audelà des présentations de surface, notamment face aux problèmes médico-pédagogiques inhabituels. Ils apportaient un feedback qui portait à la fois sur la pertinence des propositions du résident et sur le processus suivi par ce dernier pour aboutir à ces propositions. Leur feedback était davantage constructif et personnalisé. Si le temps manquait, ils proposaient de revoir le résident à une date ultérieure pour un entretien plus approfondi. De tels superviseurs étaient peu nombreux.

Un tutorat personnalisé complétait la supervision courante. Un superviseur attitré y suivait le résident sur l'ensemble de son stage. Cela aboutissait à des entretiens pédagogiques spécifiques. La démarche intellectuelle du résident et ses difficultés dans le déroulement de la prise en charge du patient y étaient explicitées. L'exploration des connaissances utilisées par le résident, et du pro- cessus par lequel il développait sa représentation du problème médical rencontré, était conduite à cette occasion. Cette exploration donnait lieu à un feedback personnalisé et ciblé. L'habileté du superviseur à la conduite d'entretien y prenait une place importante.

Les outils utilisés lors des entretiens pédagogiques s'inspiraient de la méthode réflexive de Schön $\left(i n^{[12]}\right)$ et de l'approche «CalgaryCambridge » $\left(\right.$ in $\left.^{[20]}\right)$. L'expérience acquise dans le maniement de ces techniques et l'expérience pédagogique étaient inégales selon les superviseurs. Certains ne les utilisaient pas.

\section{Une piste pour apporter des solutions aux difficultés rencontrées : l'entretien d'explicitation}

\section{Principes généraux de l'entretien d'explicitation}

L'entretien d'explicitation est une méthode développée par Vermersch, pour faciliter et provoquer le «réfléchissement» des savoirs tacites. Cette méthode aide le sujet à prendre conscience de sa démarche dans l'action et à la corriger le cas échéant ${ }^{[4,5]}$. Elle évite les biais mentionnés précédemment. L'intervieweur doit être spécifiquement formé à ce type d'entretien car l'aide à la verbalisation nécessaire pour parvenir à l'explicitation souhaitée est contre-intuitive. Le retour réfléchissant sur soi est déstabilisant pour l'interviewé, ce qui nécessite une gestion spécifique.

Pour explorer les savoirs tacites dans l'action, l'entretien d'explicitation aide le sujet à établir a posteriori un rapport spécifique avec le déroulement de son action; c'est la «position de parole incarnée ». Installé dans cette position de parole, le sujet devient capable de revivre son action et d'en décrire le vécu. Le savoir tacite recherché se découvre dans ce vécu d'action. L'obtention de cette position de parole passe par un guidage spécial qui vise à faire décrire les perceptions dans l'action et les gestes réalisés ensuite :

- concernant la prise d'information : «qu'as-tu $v u$. . entendu. . . senti... ? etc. $»^{[4,5]}$;

- concernant la localisation : " où regardais-tu... écoutais... sentais... ? etc. » ${ }^{[4,5]}$; 
- concernant l'ordre chronologique : "par quoi as-tu commencé... qu'as-tu fait ensuite... comment savais-tu que c'était terminé ? etc. » ${ }^{[4,5]}$;

- concernant les pensées : "à quoi as-tu pensé... qu'est-ce qui t'as orienté vers cette idée? etc. »;

- concernant les gestes réalisés : «qu'as-tu fait... comment l'as-tu fait... qu'est-ce qui t'a poussé à faire ce geste? etc. ».

Les questions posées évitent le «pourquoi ?» pour préférer le «comment? : "qu'est-ce qui fait que tu as commencé par...? ", " comment savais-tu que...? », "qu'as-tu fais quand...? », etc. Les connaissances réellement impliquées dans l'action sont interpelées par les formulations de type «comment?», «qu'est-ce qui fait que? », etc. Les formulations de type «pourquoi ? » se réfèrent plutôt à l'opinion du sujet sur la tâche qu'il fait, et sont à haut risque de susciter les biais cités ci-avant ${ }^{[5]}$. Ainsi, il arrive que le sujet adopte un mode de verbalisation différent de la «position de parole incarnée », la «position de parole d'interlocution ».

Vermersch s'est aidé de la programmation neurolinguistique pour distinguer les types de position de parole et pour guider le sujet pendant l'explicitation. Il n'est pas possible d'aborder ici l'ensemble de ce que cette approche a apporté à l'entretien d'explicitation. Brièvement, la «position de parole incarnée » se reconnaît au comportement non verbal du sujet pendant la verbalisation, et au contenu de cette dernière : débit de parole ralenti, entrecoupé de pauses, regard qui décroche ou se fige, comportement verbal accordé au non verbal, contenu descriptif de la verbalisation. La «position de parole d'interlocution » se reconnaît à un débit fluide, sans pause, un regard vers l'intervieweur, mobile, un contenu générique ou abstrait : "je fais toujours comme ça», "en général, ça ne me pose pas de problème », "il faut commencer par ${ }^{[5]} .$.

Parmi ses emprunts à la programmation neurolinguistique, l'entretien d'explicitation utilise quatre principales références : 1) l'importance du rythme et du ton de la voix de l'interviewé et les efforts nécessaires pour s'y ajuster; 2) la synchronisation posturale et la reprise gestuelle ; 3) l'importance du comportement oculaire, du vocabulaire sensoriel et de l'ajustement à ces derniers; 4) l'analyse de la forme de la verbalisation.

En imitant discrètement le comportement rythmique et tonal adopté par l'interviewé dans sa verbalisation, puis en le ralentissant et en l'adoucissant, on peut le guider subtilement vers la « position de parole incarnée ». L'adoption discrète de postures similaires à celles de l'interviewé et l'attention portée aux gestes qui accompagnent ses propos facilitent la communication. Le repérage de certains comportements oculaires du sujet et des références sensorielles récurrentes dans ses verbalisations (visuelles, auditives, kinesthésique, etc.), informe l'intervieweur sur son mode d'encodage sensoriel privilégié. L'interpellation de l'interviewé dans ce registre facilite l'explicitation. Enfin, l'analyse de la forme de la verbalisation, permet le repérage de structures linguistiques significatives d'opérations mentales implicites dans l'action ${ }^{[4,5]}$. Ces structures en sont les manifestations de surface dans la verbalisation : on parle d'indicateurs d'implicites. Ils sont de trois principaux types : indicateur d'omission, indicateur de généralisation, indicateur de distorsion.

D'autres repères facilitent l'entretien d'explicitation $^{[4,5]}$. Ainsi, l'analyse de la tâche permet d'en connaître les détails avant l'entretien, définissant un cadre de référence et optimisant le repérage de séquences éventuellement manquantes (domaine de connaissance,...). Par ailleurs, la connaissance préalable et détaillée de la tâche à superviser illustre l'intérêt de la supervision par un expert de contenu. Ensuite, les catégories indépendantes du contenu de l'action donnent un cadre de référence en dehors de toute expertise de contenu. Toute action humaine peut être décrite par ces catégories. Elles constituent des repères permanents dans l'écoute d'un déroulement d'action. C'est l'exemple du cadre temporel et causal. Toute action a un début, des séquences, une fin. Les catégories d'ordre temporel se rapportent aux questions : «par quoi as-tu commencé ?», «comment savais-tu par quoi il fallait commencer? », « et ensuite qu'as-tu fait? », « comment savais-tu qu'il n'y avait plus rien à faire ?», etc. 
Les catégories d'ordre causal se rapportent souvent à des contraintes d'ordre dans l'action, du type : «action A impossible si action B non faite». Hors de toute spécificité de contenu, la non faisabilité d'une action suite à l'absence d'une action antérieure se retrouve dans la vie quotidienne. Il tient alors du bon sens de repérer les séquences manquantes. Quoique simpliste, ce genre de catégories peut servir de repère, mais est souvent oublié dans les démarches d'explicitation.

La mise en œuvre de l'entretien d'explicitation dans la supervision clinique suppose d'autres règles. L'entretien porte sur une tâche réelle et spécifiée, son objet est expliqué à l'étudiant. Il s'initie avec la même consigne d'un candidat à l'autre, comme une question simple et ouverte. L'étudiant est systématiquement informé qu'il sera supervisé, par qui et de quelle manière. Il est informé des qualités professionnelles et des fonctions académiques du superviseur, de l'exploitation qui sera faite des données de l'entretien. L'attitude du superviseur restera neutre quant au contenu des verbalisations produites. Cela n'empêche pas la convivialité. Le succès de l'entretien d'explicitation est dépendant de la qualité de la relation établie avec le supervisé, du climat de confiance qui en procède.

\section{Les indicateurs d'implicites}

Les exemples de verbalisations suivantes sont inventés pour illustrer nos propos, selon des modèles donnés par Grinder et Bandler ${ }^{[23]}$. Il ne nous a pas été possible d'illustrer chaque sous-type d'indicateurs.

\section{Les indicateurs d'omission (délétion)}

$\mathrm{Ce}$ sont des structures linguistiques incomplètes amenant l'expert à soupçonner un non-dit : les verbes incomplètement spécifiés, les adjectifs qualificatifs, les adverbes, les comparatifs et superlatifs, les opérateurs modaux de possibilité ou de nécessité. Voici une formulation comportant un verbe incomplètement spécifié : "Il a juré qu’il ne supportait pas ». On reconnaîtra les omissions correspondant à ce verbe et on tendra, soit à remplacer l'information manquante par des suppositions, soit à l'élucider par des questions : «Supporter quoi?». Le verbe impliqué ne se suffit pas à lui-même pour informer complètement sur son objet : il appelle un complément, non dit dans la verbalisation d'origine. Il s'agit d'un indicateur de type «omission». Voici une autre formulation comportant un adjectif qualificatif : "Je me méfie des plaies douteuses». Cet adjectif apporte une information sur le nom «plaie » qui procède d'un jugement de valeur implicite. D'où la question : "Qu'est-ce qui fait dire que cette plaie est douteuse? ». Les adverbes suivent une logique analogue aux adjectifs. Les comparatifs et les superlatifs comportent des implicites relatifs aux référentiels auxquels se rapporte la comparaison formulée. Les opérateurs modaux expriment soit une nécessité (ou une obligation), soit une possibilité (ou une impossibilité). L'implicite réside dans le mobile du jugement de nécessité (d'obligation) ou de possibilité.

\section{Les indicateurs de généralisation}

Ce sont des structures linguistiques exprimant une représentation mentale sans index référentiel précis. Elles révèlent l'extraction d'un modèle général à partir d'expériences objectives dont les détails sont omis. On y retrouve les verbes incomplètement spécifiés, les quantificateurs universels («tout», « souvent », « jamais », « toujours », « en général », etc.), les prédicateurs de symétrie, les «équivalences ». Soit une formulation avec un quantificateur universel : $(X)$ «Mme M. entre toujours en conflit avec $m o i »$. L'index référentiel omis dans cette généralisation est un contre-exemple à l'affirmation : «toujours »; l'implicite est là. Les prédicateurs de symétrie s'impliquent dans des verbalisations exprimant une relation entre personnes. Ils sont du type logique : « $\mathrm{A}$ agit sur $\mathrm{B}$ et $\mathrm{B}$ agit sur $\mathrm{A}$ ». Ces structures ont pour particularité d'omettre souvent la seconde séquence (B agit sur $\mathrm{A})$. D'où une image de relation dont le contrôle échappe au deuxième protagoniste : l'implicite est là. Par la représentation partielle : «A agit sur $\mathrm{B} »$, on crée une image d'inexorabilité de l'action de A sur B. La formulation $(X)$ contient aussi un prédicateur de symétrie. Abordons le cas des «équivalences ». Cette verbalisation contient une équivalence : «Mlle L. a une personnalité hystérique... Elle a toujours matière à se plaindre et 
tient le corps médical en échec ». On y voit la structure suivante : $\left[\right.$ proposition $\left.n^{\circ} 1\right]+[\ldots]+[$ proposition $\left.{ }^{\circ} 2\right]$. Cette structure implique souvent une équivalence tacite entre [proposition $\mathrm{n}^{\circ} 1$ ] et [proposition $\mathrm{n}^{\circ} 2$ ]. À l'oral, ce type de verbalisation se présente par l'énoncé d'une première proposition orale, puis une pause, puis une seconde proposition sans lien explicite avec la première. Néanmoins, l'auditeur comprend intuitivement que ce lien existe : l'implicite est là.

\section{Les indicateurs de distorsion}

Ce sont des structures linguistiques exprimant une réappropriation subjective d'expériences vécues. Y figurent les présuppositions, les nominalisations et les différents types de perversions sémantiques ${ }^{[24]}$.

Certaines propositions renvoient à d'autres, implicites, sans lesquelles elles ne sauraient être comprises. La compréhension des premières nécessite l'intuition des secondes : il s'agit de présuppositions. La proposition $(Y)$ suggère la présupposition (Ybis) : "S'il avait écouté mes explications, il n'aurait pas arrêté son traitement» => «Il n'a pas écouté mes explications ».

Une nominalisation est la transformation d'un verbe en nom. D'où une impression de fixité ou d'irréversibilité des conséquences d'une action. Mais, toutes les nominalisations ne cachent pas une telle notion. Le cas échéant, la suite de l'entretien permet de vérifier s'il y a implicite d'irréversibilité, ou non. Cette proposition contient une nominalisation : «Je regrette ma décision d'avoir changé son traitement ». Il s'agit de la nominalisation du verbe «décider». Les implicites peuvent être l'irréversibilité de cette "décision», et ce qui conditionne cette irréversibilité. Certaines perversions sémantiques suivent une logique similaire, telles les perversions de relation causale. Les nominalisations peuvent aboutir à ces perversions, c'est-à-dire l'altération de la représentation subjective des relations de cause à effet. Des exemples sont retrouvés dans la gestion des relations entre personnes. Cette perversion amène l'un des protagonistes à prêter à un tiers la faculté d'agir sur lui sans possibilité de contrôle. La réaction de l'individu à l'action de son interlocuteur n'est pas présentée comme subjective, mais comme impérative, inexorable, suite aux actes du vis-à-vis. D'où la déresponsabilisation du sujet dans sa relation à l'autre. L'implicite est que le sujet perçoit ses réactions comme irrépressibles, il en attribue l'entière responsabilité à son entourage. Les verbalisations exprimant cette distorsion expriment une réaction à quelque chose. La formulation suivante peut dissimuler ces distorsions : "Ma femme me déprime».

\section{Discussion}

Les constats effectués lors du stage clinique dans le cadre de notre observation participante rejoignent ceux de la littérature ${ }^{[6-13]}$. L'entretien entre le résident et l'enseignant prend une place importante dans la supervision clinique mais n'exploite pas suffisamment les opportunités de formation du résident. Il joue un grand rôle dans l'appréciation de la compétence et des difficultés du résident. Or, la formation des superviseurs aux techniques permettant d'optimiser le temps d'entretien et d'éviter les biais qui s'y rapportent est inégale d'un individu à l'autre.

Les biais faussant la supervision au cours d'un entretien se rapportent souvent à des problèmes de communication. Voici un exemple typique : l'étudiant qui se présente au superviseur a en tête une représentation subjective de son travail. Il va transmettre son image de son travail. Mais, le superviseur veut connaître la manière dont un étudiant, engagé dans un acte médical spécifique, a traité les informations qui se présentaient à lui pour parvenir à un résultat. La confrontation de ces deux positions différentes, notamment dans un contexte de stress, facilite la confusion et peut amener le superviseur à entendre comme acte réel une verbalisation qui n'est qu'une opinion sur l'action. Il y a un risque notable de contamination du rapport clinique objectif par la représentation subjective que l'étudiant a de son travail ; cette contamination est une source d'erreur. Il importe donc de réfléchir aux manières d'éviter ce piège lorsqu'on ne maîtrise par les outils d'entretien adéquats. 
L'approche de «Calgary-Cambridge » et les travaux de Schön invitent à développer des approches pédagogiques constructivistes et collaboratives entre enseignants et étudiants. Ils suggèrent que la prise en considération des processus d'apprentissage ou professionnel améliore la performance pédagogique et la satisfaction des parties prenantes. Ils ciblent la transmission d'un «savoir pratique », d'autant plus délicate que ce savoir est souvent inconscient et d'autant plus importante qu'il conditionne le succès ou l'échec. De telles approches sont cohérentes avec les recommandations retenues dans la littérature quant à l'entretien avec l'étudiant ${ }^{[6,8,10,11]}$ et quant à la communication adaptée au contexte de soins primaires ${ }^{[15-19]}$. Cependant, elles ne permettent pas de provoquer spécifiquement le «réfléchissement» des savoirs tacites, et donc n'aboutissent pas totalement à leur ré-appropriation consciente par l'étudiant. Cette ré-appropriation est pourtant le début de l'élaboration d'un savoir expérientiel conceptualisé, enseignable, expliquant «comment on fait» pour réussir (d'après ${ }^{[4]}$ ).

La littérature issue de la psycho-ergonomie propose, selon nous, une approche qui intègre de nombreuses techniques communicationnelles décrites dans l'approche «Calgary-Cambridge », et dont les bases conceptuelles recoupent les idées développées par Schön : il s'agit de l'entretien d'explicitation formalisé par Vermersch ${ }^{[4,5]}$. Ses apports originaux consistent en une conduite d'entretien spécifique, destinée à provoquer la rappropriation consciente du «savoir dans l'action». Par cette réappropriation, l'interviewé accède à la compréhension du «comment il fait ». L'intérêt est que, lorsque ce «faire» aboutit à un échec, son « réfléchissement» devient une opportunité de comprendre ses erreurs et de développer des procédures améliorées. Conduit par un expert de la tâche à apprendre, nous pensons que cette approche peut devenir un outil pédagogique puissant. De plus, cette méthode emprunte à la programmation neurolinguistique de nombreux repères d'écoute, permettant de mieux comprendre «de quoi on parle», ainsi que des techniques permettant de mieux s'accorder sur le contenu recherché : en d'autres termes, on évite les biais fréquemment associés aux entretiens consécutifs à l'action.

Nous pensons, en outre, que l'amélioration de l'efficacité pédagogique de l'entretien passe par la limitation des biais venant d'une planification inadaptée. L'entretien pédagogique devrait se dérouler immédiatement après chaque entrevue avec le patient. On éviterait ainsi les malentendus, le délai de verbalisation des tâches réalisées, et la difficulté due à la remémoration d'actes lointains. De plus, dans les situations où l'étudiant s'est senti en difficulté sans pouvoir accéder facilement aux connaissances manquantes, le temps pourrait accroître le risque de voir combler ses lacunes par des théories naïves et sécurisantes «auto-produites » sur les raisons de ses choix. Lorsque la supervision ne peut être que différée, l'enregistrement de la consultation sur vidéo pourrait faciliter à distance l'explicitation du raisonnement dans l'action. Sous guidance adaptée, la vidéo pourrait offrir à l'étudiant le moyen de se reconnecter à l'action et de se remémorer ses impressions et pensées du moment.

Il convient cependant de souligner quelques limites potentielles de notre proposition : elles sont à la fois de nature conceptuelle et de nature opérationnelle. Au plan conceptuel, les liens que nous avons tissés entre les travaux de Schön, l'approche formalisée dans le modèle de "Calgary-Cambridge » et les données que nous avons extraites de la littérature en psycho-ergonomie, résultent essentiellement d'une argumentation théorique, mettant en perspective plusieurs convergences que nous percevons entre ces différents courants. Un tel cadre conceptuel mériterait cependant d'être approfondi et confronté systématiquement à la vérification par des travaux de recherche empirique ultérieurs. Au plan opérationnel, la maîtrise des approches communicationnelles et pédagogiques que nous avons présentées, nécessite une formation approfondie. Cela peut limiter la généralisation de leur application à la supervision clinique. Une telle formation préalable est incontournable dans le cas de l'entretien d'explicitation. De plus, la démarche d'explicitation nous paraît exiger un minimum de 20 minutes, pour 
les tâches les plus simples. En dehors d'une expérience pratique suffisante de cette approche, il nous paraît difficile de l'appliquer dans le cours de la supervision de routine. Ses champs d'applications en formation médicale restent à définir; nous pensons qu'elle s'intégrerait volontiers dans un tutorat personnalisé. Des formations aux techniques d'aide à l'explicitation sont proposées en France dans le cadre du GREX, groupe de recherche sur l'explicitation.

\section{Conclusion}

Notre travail souligne l'importance d'une formation sérieuse aux approches communicationnelles et aux techniques d'investigation des procédures impliquées dans l'apprentissage et dans l'action professionnelle. En son absence, la qualité de la prise en charge pédagogique de l'étudiant, au cours de l'entretien qui accompagne la supervision clinique, ou des tutorats intégrés à la formation, nous paraît non optimale. Nous pensons que les approches communicationnelles et pédagogiques, inspirées de l'approche Calgary-Cambridge et des idées de Schön, améliorent la formation du résident. En assumant les limites évoquées précédemment, nous suggérons que l'entretien d'explicitation est susceptible d'y ajouter une aide à l'élaboration des savoirs expérientiels et à l'auto-critique, qui peut se prolonger par le développement d'aptitudes à l'autoformation. Manié par un expert, nous pensons qu'il pourrait devenir un outil pédagogique puissant dans le cadre d'un tutorat personnalisé.

\section{Remerciements}

Nous remercions : Monsieur Pierre Vermersch, psycho-ergonomiste (CNRS, GREX, Paris, France) pour ses commentaires et les informations transmises à propos de l'entretien d'explicitations ; Monsieur Daniel Liévin, ergonomiste (laboratoire de psychologie de l'homme au travail, INRS, Vandœuvre lès Nancy, France) pour les documents et les informations fournis à propos de l'entretien d'explicitation; Madame Marie-Thérèse Lussier, psychologue et médecin de famille (cité de la santé, Laval, Québec, Canada) pour les informations transmises concernant la méthode «CalgaryCambridge » et les techniques de communication professionnelle en santé.

\section{Contributions}

Thierry Liévin a proposé la problématique du travail, organisé et mis en œuvre la logistique du stage d'observation, conçu le protocole de recherche bibliographique, effectué le recueil des données, la synthèse des résultats et rédigé le manuscrit. Marquis Fortin a proposé le sujet et la problématique, est intervenu en tant qu'expert de contenu et a participé à la relecture du manuscrit. Bernard Millette a contribué à l'organisation et la mise en œuvre logistique du stage d'observation, est intervenu en tant qu'expert de contenu et a participé à la relecture du manuscrit. Alain Aubrege est intervenu en tant qu'expert de contenu et a participé à la relecture du manuscrit. Jean-Dominique de Korwin a contribué à l'organisation formelle du travail, à la synthèse des résultats et à la relecture du manuscrit.

Cet article a été élaboré à partir du travail de thèse pour le diplôme d'état de docteur en médecine de Thierry Liévin : bilan d'une année de stage de résident dans une unité de médecine familiale canadienne; expérimentation et analyse des méthodes pédagogiques; perspectives pour l'enseignement de la médecine générale en Franceuniversité Henri Poincaré de Nancy, septembre 2003. Il a fait l'objet d'une communication orale lors du «troisième forum international francophone de pédagogie médicale », les 3 et 4 juin 2004, à Sherbrooke, Québec, Canada. 


\section{Références}

1. Kilminster S, Cottrell D, Grant J, Jolly B. AMEE Guide No 27: Effective educational and clinical supervision. Med Teach 2007;29:2-19.

2. Kilminster S, Jolly B. Effective supervision in clinical practice settings: a litterature review. Med Educ 2000;34:827-40.

3. Martineau B, Girard G, Boulé R. Interventions en supervision directe pour développer la compétence du résident : une recherche qualitative. Pédagogie médicale 2008;9:19-31.

4. Vermersch P. L'entretien d'explicitation. Collection Pédagogies, Paris : ESF Editeur, 1994.

5. Vermersch P. Questionner l'action : l'entretien d'explicitation. Psychologie Française 1990;35:227-35.

6. Irby M. How attending physicians make instructional decisions when conducting teaching rounds?, Acad Med 1992;67:630-8.

7. Irby DM. Teaching and learning in ambulatory care settings: a thematic review of the literature, Acad Med 1995; 70:898-931.

8. Irby D.M. What clinical teachers in medicine needs to know. Acad Med 1994;69:333-42.

9. Knudson MP, Lawler FH, Zweig SC, Moreno CA, Hosokawa MC, Blake RL. Analysis of resident and attending physician interactions in family medicine, J Fam Pract 1989;28:705-9.

10. Lesky LG, Hershman WY. Practical approaches to a major educational challenge: training students in the ambulatory setting. Arch Intern Med 1995;155:897-904.

11. Poole AD, Sanson-Fisher RW. Long-term effects of empathy training on the interview skills of medical students. Patient Couns Health Educ 1980;2:125-7.

12. Shapiro J, Talbot Y. Applying the concept of the reflective practitioner to understanding and teaching family medicine. Fam Med 1991;23:450-6.

13. Woolliscroft JO, Schwenk TL. Teaching and learning in the ambulatory setting, Acad Med 1989;64:644-8.

14. Williamson HA, Glenn JK, Spencer DC, Reid JC. The development of clinical independence: resident-attending physician interactions in an ambulatory setting, J Fam Pract 1988;26:60-4.
15. Beck RS, Daughtridge R, Sloane PD. Physician-patient communication in the primary care office: a systematic review. J Am Board Fam Pract 2002;15:25-38.

16. Poole AD, Sanson-Fisher RW. Long-term effects of empathy training on the interview skills of medical students. Patient Couns Health Educ 1980;2:125-7.

17. Vegni E, Martinoli M, Moja EA. Improving patient-centred medicine: a preliminary experience for teaching communication skills to Italian general practitioners. Educ Health 2002;15:51-7.

18. Florenzano R, Altuzarra R, Carvajal C, Weil K, Dorr A, Fullerton C, Gottlieb B, Baeza H, Ramirez L, Barcos P, Cerda X, Donoso R. Improving the quality of clinical interview teaching: evaluation of an intervention with medical students [Article in Spanish]. Rev Med Chil 2000;128:294-300.

19. Makoul G, Schofield T. Communication teaching and assessment in medical education: an international consensus statement, Netherlands Institute of Primary Health Care. Patient Educ Couns.1999;37:191-5.

20. Richard C, Lussier MT. La communication professionnelle en santé. Québec (QC) : Éditions du Renouveau Pédagogique, 2005.

21. Schön DA. The Reflective Practitioner: How Professionals Think in Action. New-York: Basic Books, 1983.

22. Caverni JP. La verbalisation comme sources d'observables pour l'étude du fonctionnement cognitif. In : Caverni JP, Bastien C, Mendelsohn P et al. (Eds.) Psychologie cognitive : «Modèles et Méthodes ». Grenoble : PUG, 1988 : 253-273.

23. Grinder J, Bandler R. The structure of magic. Palo Alto (CA) : Science \& Behavior Books, 1976.

24. Charlin B. Apprendre à réfléchir dans l'action. ACEM $1997 ; 8: 2-4$

Correspondance et offprints : Thierry Liévin, Département universitaire de médecine générale, Faculté de médecine, 9 avenue de la Forêt de Haye, BP 184, 54505 Vandœuvre lès Nancy Cedex, France.

Mailto : lievin.thierry.doc@gmail.com 